\title{
Working Memory, Reading and Math Fluency in Children with the Human Immunodeficiency Virus (HIV+)
}

\author{
Kalima Kalima ${ }^{1}$, Menon Anitha ${ }^{2}$ \\ ${ }^{1}$ School of Education, University of Zambia, \\ ${ }^{2}$ School of Humanities and Social Sciences, University of Zambia
}

\begin{abstract}
In this paper, we discuss working memory, reading and math fluency in children with the Human Immune Virus (HIV). Several studies have been conducted to investigate the effects of the Human Immune Virus (HIV) on cognitive function of individuals. An investigation of the effects of the virus on reading and fluency skills of children aged 12-14 was conducted using neurocognitive subtests measuring Short-term Memory, Working Memory, Rapid Automatized Naming (RAN) while Reading and Math Fluency (as measures of academic function) were assessed using the Woodcock Johnson Test of Achievement. The results show statistically significant differences in performance between children who were HIV positive (HIV+) compared to those who were HIV negative (HIV-). While both groups showed poor Reading and Math Fluency skills, the seropositive sample had poorer performance in Working Memory, Reading and Math fluency. The findings further demonstrate significant correlations between Working Memory and the academic skills, specifically reading and math fluency. These findings open possibilities for investigating the specific contributions that Working Memory can make in improving academic competences, specifically numeracy and literacy skills, of children.
\end{abstract}

Keywords: Working memory; Reading Fluency; Math Fluency; HIV positive

\section{INTRODUCTION}

Working Memory has been used to investigate the stability of neurocognitive function in many studies. Through many investigations, working memory has been shown to affect several aspects of daily function including, and more especially, the ability to learn. Several studies have shown how HIV infection has affected neurocognitive pathways of seropositive individuals (Dawes and Grant, 2007; Vahia et al. 2006; Katherine et. al, 2000). Most of theses studies have been conducted on adult populations and few studies have focused on paediatric populations. This study was particularly important as it contributes to an investigation of the effect of HIV on a specific construct, working memory, which unlike short-term memory, requires the manipulations of stored information. The importance of this study is anchored on firstly, the fact that the study was conducted in a region with a different clad of the HIV virus and secondly an emphasis on an investigation on the specific contribution made by working memory on two academic constructs that determine the success or failure of children to progress in national examinations. Most examinations are timed such that examinees are required to store and process information accurately and rapidly in order to pass the examinations. Working memory, unlike short-term memory plays an important role in this aspect. It is on this basis that this study was undertaken to investigate the differences in working memory for seropositive children compared to a control group of seronegative children.

There has been wide-spread debate about the difference between short-term memory and working memory. Some studies have used these terms interchangeably to mean the same thing.(St-ClairThompson and Allen, 2012). There are other researchers who have argued that the two skills are different. In this paper, we take the position that the two constructs represent different skills. Shortterm memory has been defined as the capacity to store information without necessarily manipulating it. Working memory on the other hand, has been defined as the processes that are involved in first storing and manipulating information simultaneously (Cowan, 2008). 


\section{METHODS}

\subsection{Participants}

The study consisted of 75 children (40 HIV seropositive and 35 HIV seronegative) from government hospitals and schools. These are institutions that are run by government. The seropositive groups were recruited from the hospitals during their schedule review days, while the children from the schools were recruited while they schools were in session but the testing was done during the vacation. This was in order to avoid pupils missing out on learning for the duration of the test taking period. The mean age for the sample was 12.9 years (with the mean age for the seropositive group 13.1 years and 12.6 years for the seronegative sample). Parental informed consent was obtained and verbal consent from the children in the study.

\subsection{Procedure}

Each of the children was assessed in a quiet environment. The children were required to take the test over a period of 40 to 60 minutes depending on individual test taking skills. The children were tested at the hospital or school depending on whether they were the seropositive or seronegative group. The children were tested on memory skills, language, math and reading fluency. The testing was administered using the standard administration procedures for each child. It was noted during the study that some children were unable to read and this contributed to the variation in the number of children taking the different measures. There were children in the study group, as well as those in the control group who were unable to read and so did not take the fluency tests. In this paper, only the Memory, Language and achievement skills as represented by the Math and Fluency skills reported.

\subsection{Memory Tests}

There were 3 memory tasks that were assessed, Digit Recall Forward (DRF), Digit Recall Backward (DRB) and Verbal Memory (VL). These were from a variety of standardised measures.

Digit Recall: The Digit recall tasks (Forward and Backward) were from the Kaufman's Assessment Battery for Children-Second edition (KABC-II). There are 22 combinations of digits ranging from 110. The digits are arranged in random order for each item. The participants are asked to recall digits after the examiner presents the digits. In the first trial, the participants recall the digits verbatim, as the examiner presented them. In the second trial, the participants are requested to say the number presented by the examiner in reverse.

Verbal Learning: Verbal Learning was assessed using the California Verbal Learning Test (CVLT). This test measures recall and recognition of words presented over a number of times. The words are representative of everyday items that are familiar to the children. There are 15 items in this test.

\subsection{Language Tests}

Language was assessed using a subtest from the Neuropsychological Assessment Tool-Second Edition (NEPSY-II).

Random Automatized Naming: Speeded Naming subtest of the NEPSY was used to assess the participants' ability to was used to assess the language skills of the participants. This task has been shown in literature to contribute to the individuals' future success in reading.

\subsection{Reading Fluency}

This was assessed using the Woodcock Johnson Test of Achievement-Third edition (WJ-III). Participants were required to read sentences and decide whether the sentence was correct or not. The participant is given 3 minutes in which to answer as many questions as possible.

\subsection{Math Fluency}

The participants' fluency in math was also measured using the Woodcock Johnson Test of Achievement-Third edition (WJ-III). The Children were required to solve simple math problems within 180 seconds. The participant is given 3 minutes in which they are required to solve as many problems as possible. The questions are simple addition, subtraction, division and multiplication.

\section{RESUlTS AND DisCUSSION}

Sample size, means and standard deviations are for dependent and independent variables are reported in Table 1. 
Working Memory, Reading and Math Fluency in Children with the Human Immunodeficiency Virus $(\mathrm{HIV+)}$

Table1. Descriptive statistics for Working Memory, Random Automatized Naming, Reading and Math Fluency

\begin{tabular}{|l|c|c|c|c|}
\hline Variable & $\mathrm{n}$ & Mean & Standard Deviation & Range \\
\hline Age & 75 & 12.90 & .90 & $12-14$ \\
\hline Grade Level & 75 & 6.58 & .66 & $4-8$ \\
\hline Literacy and Numeracy & & & & \\
\hline Reading Fluency & 64 & 18.6 & 10.29 & $0-40$ \\
\hline Math Fluency & 75 & 31.63 & 14.79 & $1-79$ \\
\hline Verbal Learning & & & & \\
\hline Verbal Learning Immediate & 70 & 4.96 & 1.60 & $0-8$ \\
\hline Verbal Learning Delayed & 70 & 7.81 & 2.19 & $3-13$ \\
\hline Speeded Naming Completion Time (RAN) & 70 & 59.87 & 28.32 & $9-199$ \\
\hline Digit Recall & & & & \\
\hline Forward & 73 & 6.22 & 1.98 & $2-11$ \\
\hline Backward & 73 & 2.77 & 1.98 & $0-7$ \\
\hline
\end{tabular}

Note: Although the total sample size was 75, sample sizes for different tests varied. The lowest being the Reading Fluency as some of the participants stated that the cannot read and therefore could not take the test.

The data in this study yielded Kaiser-Meyers-Olkin (KMO) measure of sampling adequacy of .69 which is an acceptable value. The Bartlett's test of sphericity was also calculated and yielded statistically significant values, $x^{2}(55)=165.705, p=0.000$.

\subsection{Group Comparison}

The HIV status was associated with poorer performance in all variables that were investigated in the present study with the seropositive group performing poorly compared to the seronegative group. To test the hypothesis that seropositive group $(n=40)$ had significantly lowerscores on all variables compared to the seronegative group $(n=35)$, the independent samples t-test was performed. The results indicate that while there appears to have been differences in mean scores between the HIV+ and HIVgroups, the differences in mean scores for the variables did not reach statistical significance $(\mathrm{p}<.05)$ for all the variables under investigation, except in the Speeded Naming Time (measuring the Random Automatized Naming speed) and the Digit Recall Backward (measuring Working Memory) tests. Ttest results are presented in table 2.The mean completion time for Speeded Naming for the HIV+ group $(M=69.33 ; \mathrm{SD}=33.47)$ was significantly higher than that of the HIV-group $(\mathrm{M}=51.00 ; \mathrm{SD}$ $=15.35): t(49)=2.709 ; \mathrm{p}=0.01$. Cohen's $d=.64$, a 'medium' effect. These findings agree with those of Fellows et al (2014) who found that information processing skills in persons living with HIV were poorer for than controls who where HIV negative.

The Digit Recall Backward, as a measure of Working Memory yielded a mean score for the HIV+ group $(\mathrm{M}=2.33$; $\mathrm{SD}=1.74)$ significantly lower than the HIV- group $(3.26 ; \mathrm{SD}=1.21): t(71)=$ 2.618; $\mathrm{p}=0.01$ ) Cohen's $d=.11$ a 'minimal' effect. These findings are consistent with the findings of Martin et al, (2001) that HIV-related working memory deficits are evident and affect many different aspects of information processing in persons living with HIV. All the statistics were calculated with a confidence interval of $95 \%$.

Table2. T-test results for the dependent and independent variables

\begin{tabular}{|c|c|c|c|c|c|}
\hline & \multicolumn{3}{|c|}{ Mean (SD) } & $\mathrm{t}$ & $p$ \\
\hline & Seropositive (HIV+) & Seronegative (HIV-) & Group & & \\
\hline $\mathrm{RF}$ & $16.21(11.55)$ & $20.66(8.77)$ & $18.64(10.29)$ & -1.75 & .08 \\
\hline MF & $29.22(16.13)$ & $34.37(12.76)$ & $31.63(14.88)$ & -1.52 & .13 \\
\hline VL & $7.81(2.38)$ & $7.82(2.00)$ & $7.81(2.19)$ & .40 & 989 \\
\hline SN(time) & $69.33(33.48)$ & $51.00(15.35)$ & $60.43(27.68)$ & 2.71 & $.00 *$ \\
\hline DRF & $6.05(2.11)$ & $6.44(1.86)$ & $6.23(1.99)$ & .65 & .409 \\
\hline DRB & $2.33(1.74)$ & $3.26(1.24)$ & $2.78(1.58)$ & 2.62 & $0.01 *$ \\
\hline
\end{tabular}

Notes: RF: Reading Fluency; MF: Math Fluency; VL: Verbal Learning; SN Speeded Naming Completion time; DFR: Digit Recall-Forward; DRB: Digit Recall-Backward.

$* p=0.01$. 


\subsection{Correlations}

The Correlations matrices are shown in table 3.

The variables were correlated with each other to investigate the relationships that existed among them. There were inter-correlationships in the neurocognitive variables and the academic variables. Our main variable under investigation, working memory, reading and math fluency, were correlated with Age, Gender, Grade level, Verbal Learning and Naming Speed.

The results show that working memory was correlated with grade level $(r=.43, \mathrm{p}<.01)$; Reading Fluency $(r=.43, \mathrm{p}<.01)$; Math Fluency $(r=.52, \mathrm{p}<.01)$; Naming Speed $(r=-.54, \mathrm{p}<.01)$ and Short-Term Memory $(r=.40, \mathrm{p}<.01)$. These correlations of the variables of interest ranged from medium (.43) for Reading Fluency and to large for Math Fluency (.52).We performed a bivariate correlation of the Reading and the Math Fluency tests with the Verbal Learning test. The results did not reach statistical significance.

Table3. Correlations of variables under study

\begin{tabular}{|l|l|c|c|c|c|c|c|c|c|}
\hline & & 2 & 3 & 4 & 5 & 6 & 7 & 8 & 9 \\
\hline 1 & Age & -.19 & $.29 * *$ & -.04 & .19 & .07 & $-.24 *$ & -.08 & -.02 \\
\hline 2 & Gender & - & -.07 & .09 & .10 & .12 & -.07 & .06 & .03 \\
\hline 3 & Grade & & - & $.31^{*}$ & $.53^{* *}$ & .18 & $-.31^{* *}$ & .19 & $.43^{* *}$ \\
\hline 4 & Reading & & & - & $.54^{* *}$ & .15 & $-.63^{* *}$ & $.26^{*}$ & $.43^{* *}$ \\
\hline 5 & Math & & & & - & .20 & $-.61^{* *}$ & $.29^{*}$ & $.52^{* *}$ \\
\hline 6 & Verbal & & & & & - & -.01 & .11 & .00 \\
\hline 7 & Naming & & & & & & - & $-.31^{* *}$ & $-.54^{* *}$ \\
\hline 8 & Digit-F & & & & & & & - & $.40^{* *}$ \\
\hline 9 & Digit-B & & & & & & & & - \\
\hline
\end{tabular}

Notes: *p<.05; **p<.01

\section{Conclusion}

The study investigated the role of Working Memory in Reading and Math Fluency in HIV+ Children. The findings have demonstrated the role of working memory in good reading and math skills. The study compared short-term memory and working working memory for children who were seropositive and those that were negative with mean age of 12.9 years.

The study has shown how working memory correlates with academic skills specifically Reading Fluency and Math Fluency. The correlations was higher for math fluency(r=.43) than it was for the reading fluency $(\mathrm{r}=.52)$.

This study presents possibilities for further investigations to show how that working memory can be enhanced in children so as to improve their skills in reading and writing. Further the study has shown the significant mean differences in performance in the the Working Memory and and the speed of information processing as measured by the Speeded Naming Test from the NEPSY.

\section{REFERENCES}

Alloway T.P (2007) Working memory, reading and mathematical skills in children with developmental coordination disorder. Journal of Experimental Child Psychology.(96);20-36

Cowan P (2008) Processing limits of selective attention and working memory: Potential implications for interpreting. Interpreting. 5 (2): 117-146

Dawes S and Grant I.(2007) Neurocognitive assessment of persons with HIV disease. Handbook of Clinical Neurology, (85); 93-121

Fellows R, Byrd D and Morgello S (2014) Effects of Processing Speed on learning, memory and executive functions in people living with HIV/AIDS. Journal of Clinical Experimental Neuropsychology (36) 8, 3, 806-817

Gray C \&Kinnear P (2012) IBM SPSS 19 Made Simple. New York. Psychology Press

Katherine A, Stehbens J, Mahoney E, Sirois P, Nichols A, Bordeaux J, Watkins J, Amodei N, Hill S and Donfield S(2000) Declining Immune Function in Children and Adolescents with Hemophilia and HIV Infection: Effects on Neuropsychological Performance. Journal of

Pediatric Psychology. (25)5, 309-322) 
Martin E, Sullivan T, Reed R, Fletcher T, Pitrak D, Weddington W and Harrow M(2001) Auditory working memory in HIV-1 infection. Journal of the International Neuropsychology Society (7), 20-26

Rosenthal E, Riccico C, Gsanger K \& Jarratt E. (2006) Archives of Clinical Neuropsychology (21). $131-139$

St. Clair-Thompson H \& Allen R.(2013). Are forward and backward recall the same? A dual-task study of digit recall. Memory and Cognition 41:519-532

Vahia V.N, Bhojraj T and Creado D (2006) Neurocognitive deficits in HIV-Positive patients- two case reports: Revising current AANTF guidelines in view of revent revelation of new neurocognitive symptoms. Indian Journal of Psychiatry (48); 193-195 